\title{
Finasteride in the treatment of patients with benign prostatic hyperplasia: a review
}

This article was published in the following Dove Press journal:

Therapeutics and Clinical Risk Management

3 July 2009

Number of times this article has been viewed

\author{
Angela B Smith \\ Culley C Carson \\ University of North Carolina \\ Division of Urologic Surgery, \\ Chapel Hill, NC, USA
}

Correspondence: Culley C Carson University of North Carolina Division of Urologic Surgery, 21 I 3 Physicians Office Building, CB 7235, 170 Manning Drive, Chapel Hill, NC 27599-7235, USA

$\mathrm{Tel}+\mathrm{l}$ 919-966-2574

Fax + I 919-966-0098

Email carson@med.unc.edu

\begin{abstract}
Benign prostatic hyperplasia (BPH) is a complex and progressive disease common in aging men. While associated with bothersome lower urinary tract symptoms, it may also result in additional serious complications such as refractory hematuria, acute urinary retention, and BPH-related surgery. Medical therapy has been offered as an approach to halt this progression and perhaps reverse the pathophysiology of BPH. While alpha-blockers provide rapid relief in the form of improved flow rate, their effects may not reduce the overall risk of BPH-related complications. $5 \alpha$-reductase inhibitors were therefore introduced to affect the underlying disease process by inhibiting the enzyme which converts testosterone to dihydrotesterone, the primary androgen involved in normal and abnormal prostate growth. Through this inhibition, prostate size is decreased, thereby reducing the risk of acute urinary retention and BPH-related surgery while providing symptom control. These effects are most pronounced in men with enlarged prostates $(>25 \mathrm{~mL})$ who are at the greatest risk of disease progression. This article reviews the literature for finasteride used in the treatment of BPH and provides evidence for its efficacy, safety and tolerability, applicability for combination therapy, and considerations of its effects on prostate cancer risk.
\end{abstract}

Keywords: finasteride, $5 \alpha$-reductase inhibitor, prostatic hyperplasia

\section{Introduction}

Benign prostatic hyperplasia (BPH) is a pathologic process which may contribute to lower urinary tract symptoms in aging men. A common problem among males over 50 years, its prevalence increases with age and many longitudinal studies have demonstrated the progressive nature of the disease. ${ }^{1}$ Histologically, BPH is characterized by an increased number of both epithelial and stromal cells in the periurethral area of the prostate. There is controversy as to whether this increase is secondary to epithelial and stromal proliferation or impaired apoptosis leading to cellular accumulation. Nevertheless, it is understood that androgens, growth factors, neurotransmitters and other cell interactions play a role in the development of this condition.

While alpha-blockers provide rapid relief in the form of improved flow rate, their effects may not reduce the overall risk of BPH-related complications. $5 \alpha$-reductase inhibitors were therefore introduced to impact underlying disease by inhibiting the enzyme which converts testosterone to dihydrotesterone (DHT), the primary androgen involved in normal and abnormal prostate growth. Through this inhibition, prostate size is decreased, thereby reducing the risk of acute urinary retention and BPH-related surgery while providing symptom control. As a drug which blocks the type 2 isoenzyme submit your manuscript | www.dovepress.com

Dovepress 
of $5 \alpha$-reductase, finasteride's effects on the prostate and symptoms attributable to benign prostatic hyperplasia have been well documented in the literature. These effects are most pronounced in men with enlarged prostates $(>25 \mathrm{~mL})$ who are at the greatest risk of disease progression. This article reviews the literature for finasteride used in the treatment of BPH and examines the mode of action of finasteride, its efficacy, safety and tolerability profiles and its role in combination therapy and prostate cancer risk.

\section{Mode of action}

Normal and abnormal growth of the prostate is dependent on the presence of hormones and growth factors. The most important of these is testosterone which is converted within the prostate into its more active metabolite, dihydrotestosterone (DHT), by $5 \alpha$-reductase, a nuclear-bound steroid enzyme localized primarily in the prostatic stromal cell. This cell plays an important role in androgen-dependent prostatic growth.

Two isoenzymes have been identified, each encoded by a separate gene. ${ }^{2,3}$ The predominant enzyme in extraprostatic tissue such as the skin or liver is type $15 \alpha$-reductase. Conversely, the predominant prostatic enzyme is type 2 $5 \alpha$-reductase. This latter enzyme is critical to normal development of the prostate and hyperplastic growth later in life. type $25 \alpha$-reductase is extremely sensitive to inhibition by both finasteride as well as dutasteride, a dual type 1, type $25 \alpha$-reductase inhibitor. ${ }^{4}$ Given that several studies have demonstrated identical prostate size reduction between patients treated with finasteride as well as dual type 1, type 2 inhibitors (such as dutasteride), the role of type 1-derived DHT is unlikely to be clinically meaningful.

As a competitive inhibitor of type $25 \alpha$-reductase, finasteride prevents the conversion of testosterone to DHT, thereby lowering both serum and intraprostatic DHT levels. A study by Norman and colleagues revealed that finasteride reduced intraprostatic DHT level by $91.4 \% .^{5}$ However, finasteride does not reduce DHT levels to castrate levels since circulating testosterone is converted to DHT by the type 1 isoenzyme existing in the skin and liver.

Although the pathophysiology of clinical BPH is not considered to be dependent on prostate size, reducing the prostate's volume is thought to decrease the constant component of bladder outlet obstruction. Furthermore, men with a prostate volume of $30 \mathrm{~cm}^{3}$ or more have been shown to be 3.5 times more likely to have moderate-to-severe lower urinary tract symptoms and acute urinary retention. ${ }^{6}$ Therefore, by actively decreasing the size of the prostate through reduction of intraprostatic $\mathrm{DHT},{ }^{7}$ finasteride arguably plays an important role in the reduction of longterm risk of progression.

\section{Efficacy Prostate volume, BPH symptoms and $\mathrm{BPH}$ progression}

The effects of finasteride on prostate size have been studied extensively with maximal reduction of prostate volume achieved within 6 months. ${ }^{8}$ Since the mechanisms underlying finasteride's positive effects on prostate outcomes are thought to be mediated by volume reduction, one can assume that subjects with larger prostates may achieve greater benefit. Therefore, the majority of randomized, placebo-controlled trials have evaluated subjects with larger prostates which creates difficulty in generalizing to a typical patient, with normal-sized prostate. ${ }^{9}$

The first multicenter, randomized, double-blind, placebocontrolled clinical trial investigating the efficacy of finasteride was performed by Gormley and colleagues in 1992 and is often referred to as the North American Finasteride Trial. ${ }^{8}$ As mentioned above, the baseline prostate volumes in both the placebo and experimental groups (measured by transrectal ultrasound) were quite large $\left(60 \mathrm{~cm}^{3}\right)$, limiting its application to men with more typical size prostates. Eight hundred and ninety-five subjects with $\mathrm{BPH}$ were randomized to receive placebo or 1 or $5 \mathrm{mg}$ of finasteride for 1 year. The primary outcome measures consisted of a modified Boyarsky symptom score and peak flow rate, although measurements of prostate volume were also recorded as a secondary outcome. The modified Boyarsky symptom score, originally described by Boyarsky and colleagues ${ }^{12}$ and modified and validated by Bolognese et $\mathrm{al}^{13}$ was computed as the total sum of scores of nine symptoms: decreased urinary stream, dribbling, interruption in stream, hesitancy, feeling of incomplete emptying, straining to initiate flow, urgency, incontinence, and dysuria. Results demonstrated a mean percentage change in symptom score at 12 months of $-2 \%, 9 \%$, and $21 \%$ in the placebo, $1-\mathrm{mg}$, and 5-mg finasteride groups, respectively. These results were statistically significant when comparing placebo and 5-mg finasteride groups but not for the 1-mg group. Mean percentage changes in peak flow rate were $8 \%, 23 \%$, and $22 \%$ while the mean percentage changes in prostate volume were $-3 \%,-18 \%$, and $-19 \%$ respectively. A secondary analysis attempted to correlate symptom score improvement with reduction of prostate size. However, this did not prove to be dose-dependent, suggesting that the efficacy of finasteride may not be exclusively mediated by the reduction of prostate volume. 
Following this initial study, a second report was published in 1993, termed the International Finasteride Study. ${ }^{10}$ Known as the Finasteride Study Group, these researchers reported another multicenter, randomized, double-blind, placebocontrolled clinical trial which described the effect of finasteride on symptom score, peak flow rate, and prostate volume. Measured via transrectal ultrasound, the mean prostate volume here was also relatively large $\left(47 \mathrm{~cm}^{3}\right)$, including only prostates above $30 \mathrm{~cm}^{3}$. Results were in agreement with the North American Finasteride Trial, demonstrating a prostate volume reduction of $22 \%(P<0.001)$, increased peak flow rate by $1.7 \mathrm{~mL} / \mathrm{sec}(P<0.025)$ and improved symptom score by 3.3 points $(P=0.005)$.

While the above studies were favorable with regard to peak flow rate, prostate size reduction, and symptom score, they also demonstrated bias toward larger prostates, which presents difficulty in generalizing to a more typical patient population. The first study to examine the efficacy of finasteride on smaller prostate size was performed by Andersen and colleagues in $1995 .{ }^{11}$ Unlike prior studies, the mean prostate size in this study was approximately $40 \mathrm{~mL}$. Seven hundred and seven patients were maintained on either placebo or finasteride for 2 years. Primary outcome measures included a modified Boyarsky symptom score, peak flow rate and prostate volume, and these were examined at both 12 and 24 months. The group mean difference from placebo was statistically significant but the mean change was less than that shown in the both the North American Finasteride Trial as well as the International Finasteride Study. Time-dependent symptom score changes demonstrated a placebo response which returned to baseline by year 2 whereas the finasteride response remained effective throughout the study time period. Mean differences between symptom scores of the placebo and finasteride groups, however, were not markedly different, with -0.3 and 0.6 units change in symptoms between 12 and 24 months, respectively. However, when assessing mean change of symptom score between baseline and month 24 , a more dramatic change of symptom score is demonstrated in the finasteride group (2 units) compared to placebo ( 0.2 units) $(P<0.01)$. Although statistically significant, one may question the clinical application of a small difference between the two groups.

In 1994, Stoner and colleagues took advantage of the previously described large sample sizes of both the North American Finasteride Trial and International Finasteride Study, offering these subjects an open-label extension after completing 1 year of randomized therapy. ${ }^{14}$ As a long-term, 3-year efficacy analysis, 543 subjects were included in the study, although only $55 \%$ of patients continued to participate through the trial's 3-year duration (297 subjects). Two important findings emerged from this study. First, the largest change in symptom score, prostate volume, and peak flow rate occurred between 12 and 18 months, coinciding with the transfer of blinded to unblinded treatment. Second, after 18 months of therapy, the previously described changes remained stable, suggesting durability of response. Hudson and associates took this one step further, extending the analysis to five years and confirming durability of response for this time period as well. ${ }^{15}$

Another long-term study, the Proscar Long-Term Efficacy and Safety Study (PLESS), represents the double-blind, randomized, placebo-controlled study with the longest follow-up assessment to date in the medical therapy of BPH. ${ }^{7}$ During this 4-year study, 3040 men with moderate-to-severe urinary symptoms were randomized to either $5 \mathrm{mg}$ of daily finasteride or placebo. Mean prostate size was $55 \mathrm{~cm}^{3}$ in this study (measured via transrectal ultrasound, similar to prior studies), suggesting a bias toward subjects with larger prostates. Symptom scores, urinary flow rate, and outcome events were assessed every four months. A quasi-AUA (American Urological Association) symptom score was used with a mean change between placebo and treatment of 2.0 symptom units. Mean increased peak flow rate and size reduction were $1.7 \mathrm{~mL} / \mathrm{sec}$ and $32 \%$ respectively for those in the active finasteride group. Similar to Andersen's study, this confirmed durability of response as well as modest efficacy. Subjects within the finasteride group also demonstrated increased flow rates $(P<0.001)$, reduced prostate volume $(P<0.001)$ and a larger mean decrease in symptom score $(P<0.001)$ when compared to placebo. Of note, the symptom score was a self-administered questionnaire different from the now-accepted American Urological Association symptom score (as this was not yet created). However, the authors did attempt to adjust the symptom score sum to fit the AUA symptom scale, noting that they utilized a "quasi-AUA score."

Compared to prior randomized controlled trials, PLESS additionally evaluated the incidence of other outcome measures including acute urinary retention and surgical intervention for BPH. Results demonstrated a risk reduction of 55\% in men in the finasteride group with surgical intervention required in $10 \%$ of those in the placebo group and $5 \%$ in the finasteride group $(P<0.001)$. Acute urinary retention was also decreased in the finasteride group by $57 \%$ with $7 \%$ of the placebo and only $3 \%$ of the finasteride group developing this complication $(P<0.001)$.

One important consideration for this study involves the inclusion criteria, which enrolled men with moderateto-severe symptoms as well as an enlarged prostate on digital 
rectal examination. In fact, mean prostate size was measured to be approximately $55 \mathrm{~cm}^{3}$. Therefore, caution is warranted when attempting to generalize the results of this study to men without prostatic enlargement.

As there have been a number of randomized, placebocontrolled clinical trials, Boyle and associated undertook a meta-analysis of six randomized, placebo-controlled clinical trials to provide a more thorough consensus. ${ }^{16}$ The most meaningful finding was the demonstration of a positive correlation between mean change in symptom score and peak flow rate with mean baseline prostate volume, indicating that studies incorporating patients with larger prostates demonstrated greater improvements, which may account for the variability of treatment effect described in the above studies. For the 2601 men included in the analysis, there was a mean symptom improvement of 1.8 points in prostate volumes $<20 \mathrm{~cm}^{3}$ compared to 2.8 points in larger prostates $\left(>60 \mathrm{~cm}^{3}\right)$ on the Quasi-AUA Scale. Improvements in peak flow rate were also demonstrated. While men with prostate volumes $<20 \mathrm{~cm}^{3}$ demonstrated improvement of $0.89 \mathrm{~mL} / \mathrm{sec}$, this was increased significantly to $1.84 \mathrm{~mL} / \mathrm{sec}$ in men with volumes $>60 \mathrm{~cm}^{3}$. As described by Boyle, approximately $80 \%$ of the variation in treatment effect between studies was attributed to differences in mean prostate size.

Offering a different perspective on finasteride efficacy, Tammela and colleagues reported on a randomized, doubleblind, placebo-controlled study which examined the effects of finasteride on men with bladder outlet obstruction who were on a waiting list for prostatectomy. ${ }^{17}$ Urodynamic evaluation was conducted in 36 men in whom mean prostate volumes in finasteride and placebo groups were 50 and $48 \mathrm{~cm}^{3}$, respectively. Mean baseline detrusor pressures at maximal flow were 126 and $115 \mathrm{~cm} \mathrm{H}_{2} \mathrm{O}$ in the finasteride and placebo groups, respectively. After 6 months of treatment, mean changes in the detrusor pressure at maximal flow were -39 and $+3 \mathrm{~cm}$ $\mathrm{H}_{2} \mathrm{O}$ in the finasteride and placebo groups, respectively. While the results were statistically significant $(P<0.01)$, the patients in the finasteride group still required surgery to relieve obstruction, and the symptom score changes were not different between the two groups. This study was extended for 4 years as an open-label extension and this revealed further improvement of detrusor pressure at maximal flow over time with only five patients requiring surgery, demonstrating that an operation could be avoided with long-term finasteride therapy. ${ }^{18}$

\section{Hematuria}

In addition to the obvious benefits of prostate volume reduction and the halt of $\mathrm{BPH}$ progression, finasteride has also been positively associated with a reduction of refractory gross hematuria secondary to BPH. Initial reports were described by Puchner and Miller in 1995 in which a case series of 12 patients were studied. ${ }^{19} \mathrm{~A}$ hematuria grading system was devised, demonstrating $92 \%$ improvement in the hematuria grade after more than 3 months of therapy with finasteride. This was reconfirmed in 1998 with a follow-up case series, in which results were extended to a mean follow-up of 3 years, demonstrating decrease in hematuria grade over this time period during finasteride therapy. ${ }^{20} \mathrm{~A}$ second case series provided by Carlin and colleagues in $1997^{21}$ led to the development of a randomized, placebo-controlled, double-blind clinical trial by Foley and coworkers in $2000 .^{22}$ Fifty-seven patients with chronic intermittent hematuria were randomized to either finasteride or placebo. Hematuria was found to recur in $17(63 \%)$ of the untreated control group but only $4(14 \%)$ in the finasteride group $(P<0.05)$. Surgery was required in $7(26 \%)$ of patients in the control group while no finasteride-treated patient required surgery for refractory hematuria. Based on these studies, finasteride is now recommended in patients with hematuria secondary to benign prostatic hyperplasia.

The exact mechanism of finasteride's beneficial effect on BPH-related hematuria is unknown, although multiple studies have investigated this question. Donohue and associates published their experience with 64 men scheduled to undergo TURP who were randomized to receive either $5 \mathrm{mg}$ of finasteride or placebo daily for 2 weeks prior to surgery. ${ }^{23}$ Sections of prostatic urothelium were stained for VEGF expression and for CD31 to assess microvessel density (MVD) (an indicator of prostatic angiogenesis in patients with hematuria secondary to BPH). Mean MVD was found to be significantly lower in the finasteride group compared to placebo $(P<0.01)$ leading to the conclusion that finasteride inhibits angiogenic growth factors which contribute to reduced vascularity and decreased $\mathrm{BPH}$-related hematuria, even as early as 2 weeks. Memis and colleagues confirmed these findings by evaluating short-term finasteride treatment on MVD. ${ }^{24}$ Similar to the findings by Donohue, mean MVD was found to be significantly lower in patients treated with finasteride compared to control patients $(P<0.05)$.

These findings were extended by evaluating finasteride's usefulness in BPH TURP candidates. Ozdal and colleagues evaluated the effect of short-term finasteride therapy on perioperative bleeding in candidates for transurethral resection of the prostate. ${ }^{25}$ Forty patients with BPH were randomized into two groups with the first group receiving $5 \mathrm{mg}$ finasteride for 4 weeks prior to surgery and the other established as 
the control. Patients who had received finasteride or any other anticoagulant in the past were excluded from the study. No significant difference in baseline age, IPSS, prostate volume, or preoperative hemoglobin or hematocrit was demonstrated. The total blood loss and bleeding per gram of resected tissue were significantly lower in the finasteride group regardless of prostate volume ( $P<0.05$ and $P<0.0001$, respectively). Furthermore, a smaller decrease in hemoglobin and hematocrit was found in the finasteride group $(P<0.0001)$. Given these findings, finasteride is recommended not only for patients with BPH-related hematuria but TURP candidates as well for 2 to 4 weeks prior to scheduled surgery.

\section{Tolerability and adverse effects}

Although the efficacy of finasteride in reducing prostate volume and improving peak flow rate and symptom score has been established, particularly for prostate volumes $>30 \mathrm{~cm}^{3}$, this must be weighed against the potential for adverse effects associated with treatment. However, several trials have demonstrated that finasteride is well tolerated with minimal side effects.

The majority of trials note that the most common side effect is sexual dysfunction and does not constitute a clinically meaningful cause of treatment withdrawal. ${ }^{7,8,11}$ For instance, the North American Finasteride Trial evaluated the withdrawal rate of patients secondary to adverse events. ${ }^{8}$ The most common side effects included decreased libido, ejaculatory disorder, and erectile dysfunction, with rates of $3.4 \%, 2.7 \%$, and $1.7 \%$ in the $5 \mathrm{mg}$ finasteride group. Despite these adverse effects, the percentage of subjects withdrawing secondary to adverse effects did not differ in the placebo and treatment groups. Similarly, the International Finasteride Trial confirmed the most common adverse effect was erectile dysfunction (reported in $4.9 \%$ in the $5 \mathrm{mg}$ group compared to $0.4 \%$ in the placebo group) $(P=0.001)$. No differences were noted in baseline or follow-up endocrine status identified in men with or without erectile dysfunction. Despite this side effect, only 1 patient out of $246(0.4 \%)$ discontinued the study because of this side effect. No other adverse clinical effects were noted in the study.

Although the most common side effect for finasteride is sexual dysfunction, the clinical relevance of this finding has recently been called into question. A recent randomized, placebo-controlled study conducted by Mondaini and colleagues suggests a "nocebo" effect (an adverse side effect that is not a direct result of the pharmacologic action of the drug). ${ }^{26}$

One hundred and twenty patients were randomized to receive finasteride $5 \mathrm{mg}$ (concealed identity) either with or without counseling on the drug's sexual side effects. Estimation of side effects was determined at 6 and 12 months using the male sexual function-4 (MSF-4) item questionnaire as well as a self-administered questionnaire. Interestingly, those patients with prior counseling reported a significantly higher proportion of sexual side effects with the incidence of ED, decreased libido, and ejaculation disorders of $30.9 \%$, $23.6 \%$ and $16.3 \%$, respectively compared to $9.6 \%, 7.7 \%$ and $5.7 \%$ in the noncounseled group.

The minimal effect of finasteride on sexual function was further confirmed by the Prostate Cancer Prevention Trial, a randomized, double-blind, placebo-controlled clinical trial which is described in further detail later in this review. ${ }^{27}$ Sexual dysfunction was studied in over 17,000 patients during a 7-year period. A multitude of questionnaires were utilized to assess sexual dysfunction, including Sexual Activity Scale score, SF-36 Mental Health Inventory-5, Physical Function, and Vitality scores and other comorbidities which may be attributable to sexual dysfunction. Mean sexual dysfunction increased in the finasteride arm from baseline by 2.11 Sexual Activity points at the end of study $(P<0.001)$ on a total scale of $0-100$. After adjusting for confounding variables, the absolute magnitude of this dysfunction decreased further. Although statistically significant, the clinical meaning of such a small change should be taken into account given that a large sample size may show statistical significance with small, clinically insignificant differences.

Other considerations have been made with regard to androgen suppression and its adverse effects. Because androgens can affect bone turnover, lipids, hematopoiesis, as well as sexual function, Amory and associates set out to determine whether $5 \alpha$-reductase inhibitors negatively impact these end points. ${ }^{28}$ Conducting a randomized, double-blind, placebo-controlled trial, 99 men between 18 and 55 years old were randomized to receive either $0.5 \mathrm{mg}$ dutasteride, $5 \mathrm{mg}$ finasteride, or placebo for 12 months. Bone mineral density was measured at baseline, after 1 year and again at 6 months after drug discontinuation. Serum markers for bone turnover, fasting low-density lipoprotein, hemoglobin and prostatespecific antigen (PSA) were measured at baseline, after 26 and 52 weeks of treatment and at 24 weeks after drug discontinuation. Results revealed no significant difference in placebo and $5 \alpha$-reductase inhibitors for any of the above mentioned markers with the conclusion that suppression of serum DHT induced by finasteride during 12 months did not adversely affect bone turnover, serum lipoproteins, or hemoglobin.

In addition to physical side effects, animal studies have also described behavioral changes with the use of finasteride, 
and case reports of finasteride-induced depression have also been reported in humans. This was formally evaluated by Rahimi-Ardabilli, in which 128 men with androgenic alopecia were prescribed $1 \mathrm{mg}$ finasteride daily. ${ }^{29}$ Study subjects completed both the Beck Depression Inventory (BDI) and Hospital Anxiety and Depression Scale (HADS) before the start of treatment and after two months of use. Results demonstrated that finasteride-treated patients had significantly increased BDI and HADS depression scores $(P<0.01)$. HADS anxiety scores were increased in the finasteride group, but this was statistically nonsignificant $(P=0.061)$. Although a randomized controlled trial specifically investigating depression and anxiety will be necessary to confirm these findings, one should prescribe finasteride cautiously to patients with a high risk of depression.

\section{Combination therapy Efficacy}

While many earlier studies assessed the efficacy of finasteride monotherapy, research into finasteride's efficacy naturally progressed toward combination therapy and its role in the medical management of BPH. The initial multi-center, randomized, double-blind, placebo-controlled combination trial was published in 1996 by Lepor and associates and is known as the Veterans Affairs Cooperative Study No. 359. ${ }^{30}$ One thousand two hundred and twenty-nine patients were randomized into one of four groups: placebo, terazosin, finasteride, and combination therapy (terazosin + finasteride). The dose of terazosin was titrated upward to $10 \mathrm{mg}$ while $5 \mathrm{mg}$ of finasteride was provided to those patients in this treatment arm. Of the 1229 randomized patients, 1007 $(82 \%)$ completed 1 year of therapy. Mean prostate volume (measured with transrectal ultrasound) in each of the four groups ranged from 36 to $38 \mathrm{~cm}^{3}$ at baseline. The mean group differences between finasteride and placebo were not statistically significant for AUA Symptom Index, Symptom Problem Index, BPH Impact Index, and peak flow rate. In contrast, the mean differences between terazosin and finasteride were highly statistically significant, in favor of the former, unequivocally demonstrating that alpha blockade is superior to $5 \alpha$-reductase inhibitors for treatment of clinical BPH over a 12-month period. The most likely explanation of the demonstrated decreased efficacy in this study compared to the prior studies with monotherapy is the decreased baseline prostate size included in the analysis. To address this concern, a sub-analysis was performed in patients with enlarged prostates $\left(>50 \mathrm{~cm}^{3}\right){ }^{31}$ Not surprisingly, results from this sub-analysis revealed a statistically significant change in both peak flow rate as well as AUA symptom score by $2.0 \mathrm{~mL} / \mathrm{sec}$ and 1 unit, respectively, compared to placebo.

The results of the VA Cooperative Study were confirmed by the Prospective European Doxazosin and Combination Therapy Trial (PREDICT) in which 1089 men were randomized equally to placebo, doxazosin, finasteride, and combination therapy. ${ }^{32}$ Daily dose of doxazosin was titrated up to $8 \mathrm{mg}$ whereas $5 \mathrm{mg}$ dosage was used for finasteride. Baseline prostate volume was similar to that of the VA Cooperative Study at approximately $36 \mathrm{~cm}^{3}$. Improvement in peak flow rate, urinary retention and AUA Symptom Score were not significantly different between placebo and finasteride whereas improvements in these parameters were highly significantly different between doxazosin or combination therapy and placebo or finasteride, demonstrating little efficacy of finasteride in men with smaller prostate sizes over 12 months of therapy.

Another multicenter, randomized, double-blind, placebocontrolled clinical trial investigated the efficacy of combination therapy with $5 \mathrm{mg}$ SR alfuzosin and $5 \mathrm{mg}$ finasteride over 6 months of follow-up. ${ }^{33}$ Similar to the prior alpha-blocker combination trials, little improvement in IPSS was observed, and at 6 months peak flow rates were not significantly different in any of the treatment groups relative to controls.

While the above clinical trials did not show a beneficial effect of $5 \alpha$-reductase inhibition, they were limited by relatively short follow-up periods, with maximal follow-up of 1 year in any given trial, thereby potentially favoring the fast-acting benefits of alpha blockers over $5 \alpha$-reductase inhibitors. Therefore, the Medical Therapy of Prostatic Symptoms (MTOPS) Study was created to address the question of long-term benefit. ${ }^{34}$ Representing the longest and largest clinical trial conducted in patients with $\mathrm{BPH}$, MTOPS evaluated whether finasteride combined with doxazosin was more effective than either placebo or monotherapy in prevention of clinical progression of $\mathrm{BPH}$ over a period of 4.5 years. Constructed as a double-blind, randomized, placebo-controlled trial, 3047 men with BPH were enrolled with a wide variety of prostate sizes as long as the serum PSA was less than $10 \mathrm{ng} / \mathrm{mL}$. Unlike prior studies, BPH progression was used as the primary endpoint, defined as an increase above baseline of at least 4 points in the American Urological Association symptom score, socially unacceptable urinary incontinence, a $50 \%$ increase in creatinine relative to baseline values, acute urinary retention, or two or more urinary tract infections within 1 year (or a single episode of urosepsis due to bladder outlet obstruction).

Subjects were randomized into four groups: placebo, $5 \mathrm{mg}$ finasteride, doxazosin $\left(\alpha_{1}\right.$ blocker $)$ titrated to $8 \mathrm{mg}$, and 
a combination of both finasteride and doxazosin. Multiple outcome measures were assessed, including maximum flow rate, prostate volume, sexual function, and quality of life. While transrectal ultrasound and digital rectal exam were used to evaluate prostate volume, the Sexual Function Inventory Questionnaire and the Short Form-36 Health Survey were used to evaluate sexual function and quality of life, respectively. Furthermore, prostate biopsies were obtained at baseline and at 5 years in $37 \%$ of volunteering study participants (results described later).

Trial results revealed significant risk reductions in both monotherapy groups, with a 39\% risk reduction by doxazosin $(P<0.001)$ and $34 \%$ risk reduction for finasteride $(P=0.002)$ compared to placebo alone. The greatest risk reduction of clinical progression, however, was demonstrated with combination therapy $(66 \%$ risk reduction) compared to doxazosin $(P<0.001)$ or finasteride $(P<0.001)$ alone. Additional markers of clinical progression such as acute urinary retention were decreased by finasteride $(P<0.001)$ and combination therapy $(P<0.001)$ but not doxazosin. Symptom scores were also improved in the finasteride $(P=0.001)$, doxazosin $(P<0.001)$ and combination therapy $(P<0.001)$ groups.

Subanalysis of the MTOPS trial further stratified patients by prostate size. ${ }^{35}$ This revealed that men with a prostate volume $>25 \mathrm{~cm}^{3}$ achieved the greatest benefit from combination therapy compared to finasteride or alpha-blocker monotherapy. Men with prostate volumes 25 to $40 \mathrm{~cm}^{3}$ were also compared to those with volumes $>40 \mathrm{~cm}^{3}$ and no difference in treatment effect with combination therapy was noted, indicating a lower threshold for prostate size with regard to treatment efficacy with combination $\alpha$-blocker and finasteride therapy.

Given that long-term follow-up demonstrates a beneficial effect of finasteride, the next logical question is whether $\alpha_{1}$-blocker therapy may be withdrawn after a defined period of follow-up. Although no randomized clinical trials have been conducted for finasteride specifically, one trial has addressed this question with the dual type 1/type 2 $5 \alpha$-reductase inhibitor, dutasteride. ${ }^{36}$ The Symptom Management after Reducing Therapy (SMART-1) study examined this question by randomizing $327 \mathrm{BPH}$ patients to $0.5 \mathrm{mg}$ dutasteride and $0.4 \mathrm{mg}$ tamsulosin for 36 weeks and $0.4 \mathrm{mg}$ tamsulosin for 24 weeks followed by dutasteride and tamsulosin matched placebo for the remaining 12 weeks. Symptoms were assessed at weeks 24 and 30 with the IPSS. Results revealed that $77 \%$ of those who switched from combination therapy to dutasteride monotherapy did so without deterioration in their symptom score, indicating that $\alpha_{1}$-blockers may be safely removed from the combination after 24 weeks of therapy. Because the mechanism of dutasteride is believed to be similar to finasteride, the results of the study may be extrapolated to the latter. However, caution should be used in interpretation of the results as six weeks may be too short to provide conclusive evidence.

\section{Tolerability of combination therapy}

Safety and tolerability with combination therapy were evaluated in the MTOPS trial. ${ }^{7}$ While dizziness and postural hypotension were the most common side effects within the doxazosin group, erectile dysfunction, decreased libido and abnormal ejaculation were the most common encountered in the finasteride subset of patients, consistent with findings from monotherapy studies. The individual adverse effects in the combination therapy group were similar to those for each drug alone with the exception of abnormal ejaculation, peripheral edema and dyspnea, which occurred more frequently in patients taking combination therapy. This adverse effect profile was confirmed with results of the PREDICT study. ${ }^{32}$

The discontinuation rate was surprisingly lower for the patients in the combination therapy (18\%) compared to monotherapy with doxazosin $(27 \%)$ or finasteride $(24 \%)$ alone in the MTOPS trial. Of note, there were four patients who were diagnosed with breast cancer who received either finasteride alone or in combination with doxazosin. Unlike the MTOPS trial, the PREDICT study revealed almost identical discontinuation rates for the doxazosin (11.6\%), finasteride (13.6\%), combination (12.6\%), and placebo (11.9\%) groups. ${ }^{32}$ Regardless of the differences in these results, the common theme suggests tolerability of both monotherapy as well as combination therapy.

\section{Role of finasteride for prostate cancer prevention and detection}

Although this review focuses on the role of finasteride in treatment of benign prostatic hyperplasia, we would be remiss to exclude its role in prostate cancer prevention and detection, an important consideration when beginning patients on finasteride therapy. Because finasteride has been shown to reduce serum PSA, several studies have investigated the extent and clinical impact of this phenomenon. Many reports have confirmed that finasteride reduces mean serum PSA levels by approximately $50 \% \cdot{ }^{37}$ Nevertheless, the effect on PSA is highly variable, and it is therefore considered useful to establish a baseline PSA level prior to initiation of 
finasteride therapy. Biopsy for PSA elevation is warranted if the patient is on finasteride therapy, but is ultimately left to the discretion of the clinician.

The PLESS provided additional information regarding the impact of finasteride on detection of prostate cancer. ${ }^{38}$ Three thousand forty men with PSA $<10 \mathrm{ng} / \mathrm{mL}$ and no history of prostate cancer were randomized in this study to receive finasteride or placebo for up to 4 years. A pre-randomization biopsy was performed to confirm a negative status. Six hundred forty-four patients (21\%) underwent biopsy. The decision to pursue a diagnosis of prostate cancer was left to the discretion of the investigator, providing a more realistic standard practice approach. The study revealed a diagnosis of prostate cancer in $4.7 \%$ of men on finasteride and $5.1 \%$ on placebo $(P=0.7)$, suggesting that finasteride does not mask the diagnosis of prostate cancer. Furthermore, elevated PSA prompted diagnosis in $35 \%$ of cases on finasteride and $34 \%$ on placebo using an upper PSA limit of $2.0 \mathrm{ng} / \mathrm{mL}$ for finasteride and $4.0 \mathrm{ng} / \mathrm{mL}$ for placebo, yielding similar sensitivity, higher specificity, and a higher likelihood ratio for finasteride when compared to placebo. The authors conclude that multiplication of the PSA by 2 and using normal ranges for untreated men seemed to preserve the usefulness of PSA in prostate cancer detection in men taking finasteride.

The largest study investigating prostate cancer prevention with finasteride was published by Thompson and coworkers in 2003. ${ }^{39}$ Aptly named the Prostate Cancer Prevention Trial, the study randomized 18,882 men $\geq 55$ years old with a normal DRE and PSA $\leq 3.0 \mathrm{ng} / \mathrm{mL}$ to treatment with $5 \mathrm{mg}$ of finasteride or placebo for 7 years. Prostate biopsies were obtained if the annual PSA (adjusted for effect of finasteride) exceeded $4.0 \mathrm{ng} / \mathrm{mL}$ or if the DRE was felt to be abnormal. Primary end point was prevalence of prostate cancer. This outcome was detected in $803(18.4 \%)$ in the finasteride group and 1147 (24.4\%) in the placebo group, indicating a $24.8 \%$ risk reduction in prevalence over seven years $(\mathrm{p}<0.001)$. However, an alarming increase in high-grade 7 or above tumors were found in the finasteride group (37\%) versus the placebo group $(22 \%)(P<0.001)$.

Because of this anxiety-provoking finding of the Prostate Cancer Prevention Trial, subsequent analyses were performed which found that finasteride biases toward improved detection of prostate cancer as well as increased accuracy in prostate cancer grading at biopsy. Lucia and colleagues assessed whether the increased high-grade prostate cancer associated with finasteride in the PCPT was due to finasteride's potential effects on tumor morphology or prostate size. ${ }^{40}$ Prostate biopsies with Gleason scores 7-10 were examined.
When possible, comparisons were made between these biopsies and sample from radical prostatectomies, examining for tumor grade and extent. Among patients undergoing prostatectomy, the finasteride-associated increase in highgrade prostate cancer at biopsy was decreased at prostatectomy ( $46 \%$ finasteride versus $39 \%$ placebo, $P=0.10$ ), suggesting that finasteride's effects on prostate volume was more likely the contributing factor for the increase in highgrade cancers in this treatment group.

Another analysis accounted for these biases by estimating the effect of finasteride on the risk of overall and high-grade prostate cancer. ${ }^{41}$ Using estimated rates of high-grade prostate cancer based on analysis incorporating grading information from radical prostatectomies in 500 subjects, the resulting estimates for high-grade cancer rates were $8.2 \%$ versus $6.0 \%$ for placebo and finasteride, respectively demonstrating a statistically significant $27 \%$ risk reduction with finasteride $(P=0.02)$. Furthermore, a second analysis included 3-month longer collection of endpoints from the Prostate Cancer Prevention Trial (PCPT), revealing bias-adjusted prostate cancer rates of $21.1 \%$ (4.2\% high-grade) in the placebo group versus $14.7 \%$ ( $4.8 \%$ high-grade) in the finasteride group, demonstrating a non-significant difference in high-grade cancers between the two groups $(P=0.12)$. These findings collectively propose caution in concluding that finasteride increases the risk of high grade prostate cancer.

Lucia and colleagues also addressed the concern that early detection by PSA and prevention by finasteride may involve biologically inconsequential tumors. ${ }^{42}$ Tumor pathology was stratified by level of PSA for men in the placebo group who underwent radical prostatectomy. Study findings demonstrated that low cutoff levels increase detection of insignificant disease but provides more likelihood for cure whereas higher cutoff levels demonstrate the opposite. Their recommendation was therefore to taylor cutoff values for PSA screening in those with finasteride, providing men with the information that finasteride may possibly decrease their risk of disease.

A Cochrane Database Systematic Review was published in April of 2008 and succinctly outlines conclusions from the PCPT ${ }^{43}$ All randomized controlled trials published after 1984 of at least 1 year duration were included in the review. Based on this review, the AUA recently released guidelines which address the role of finasteride in prostate cancer prevention. ${ }^{44}$ Asymptomatic men with PSA $\leq 3.0 \mathrm{ng} / \mathrm{mL}$ should be notified that finasteride may reduce the incidence of prostate cancer with an additional discussion of the elevated rate of high-grade cancer with potential explanations for 
this potential risk. Furthermore, these patients should be informed that no information is known beyond seven years (as this was the duration of the largest trial [the PCPT]) and information regarding the reduction of prostate cancer mortality is unknown.

\section{Cost}

Because medical therapy for BPH may be necessary for many years, the cost effectiveness of this therapy compared to other modalities such as other pharmacologic agents as well as surgical options is an important consideration. While this has been informally discussed in several randomized controlled clinical trials, formal evaluation in the literature is limited. Johansen and colleagues investigated the cost effectiveness of multiple management options for benign prostatic hyperplasia in men with moderate-to-severe lower urinary tract symptoms and enlarged prostates $>30 \mathrm{~cm}^{3}$, including finasteride, dutasteride, tamsulosin and transurethral resection of prostate (TURP). ${ }^{45}$ Using a Markov model, the clinical progression of BPH in a cohort of 1000 men was estimated over 4- and 15.5-year periods in the Norwegian health system. Transitions between BPH symptoms, acute urinary retention, TURP, prostate cancer and death were estimated from the published literature, and sensitivity analyses were performed for direct and indirect costs for patients treated with dutasteride, finasteride, tamsulosin or TURP. Total costs for each of these treatment options at 15.5 years were $€ 3,924$, $€ 4,539, € 4,946$, and $€ 6,164$, respectively. The relative order of these costs was similar at 4 years. With these findings, dutasteride was found to be the most cost-effective, followed by finasteride and finally tamsulosin and TURP.

An abstract published by Lafuma and colleagues estimated the cost-efficacy of finasteride, doxazosin, and their combination in the treatment of BPH in France. ${ }^{46}$ Using data compiled from the MTOPS study, cost records were extracted from the National Health Insurance website and costs were determined to be $€ 7,404$ for finasteride, $€ 7,314$ for doxazosin and $€ 8,206$ for the combination. Cost-efficacy ratios were determined (additional drug costs per invasive therapy avoided) and found to be similar for finasteride and doxazosin and slightly higher for those with combination therapy. The investigators therefore concluded that the costefficacy ratio of finasteride alone or in combination with doxazosin remained in the acceptable range.

Although these studies are based on international health care cost models, the MTOPS study does provide objective evidence that combination therapy may be the most cost effective approach. ${ }^{34}$ Results from the study indicate that the number needed to treat (NNT) to prevent a case of BPH progression was 13.7 and 15.0 for the doxazosin- and finasteride-monotherapy patients but only 8.4 for the combination therapy group. The NNT was decreased further to 4.7 and 4.9 when analyzing those patients with a baseline PSA $>4.0 \mathrm{ng} / \mathrm{mL}$ and prostate size $>40 \mathrm{~cm}^{3}$, respectively, suggesting that combination therapy becomes a more costeffective approach for men with enlarged prostates in any health care model.

Finally, cost-effectiveness for finasteride as a method of prostate cancer chemoprevention has recently been evaluated. Based upon data from the Prostate Cancer Prevention Trial, Svatek and associates used a Markov decision analysis model to determine lifetime prostate health-related costs (beginning at age 50) for men treated with finasteride versus placebo. ${ }^{47}$ Results revealed a cost-effectiveness ratio of US\$122,747 per quality-adjusted life-years saved (adjusted for US\$112,062 if one assumes that finasteride does not increase the incidence of high-grade tumors). However, the cost-effectiveness ratio could be less than US\$50,000 per quality of life years saved when applied to a high-risk population with cost of US\$30 per month. Therefore, the conclusion was made that finasteride is unlikely to be cost-effective when considering impact of survival differences among treated and untreated men but potentially cost-effective in high risk populations when taking into consideration the impact of quality of life.

\section{Conclusion}

Numerous multi-center, randomized, double-blind, placebocontrolled studies have supported the role of finasteride in the treatment of BPH. As monotherapy, it has been found to significantly reduce prostate volume by over $20 \%$ with additional overall treatment-related improvements in symptom score and peak flow rate. As an added benefit, finasteride has proven efficacious in the management of refractory hematuria secondary to BPH and in preparation for TURP. Several studies have additionally investigated its role in combination therapy, and while short-term studies did not report a significant benefit, one long-term study revealed significant advantages with combination therapy with alpha-blockers to prevent BPH progression events such as acute urinary retention and need for BPH-related surgery. Treatment with finasteride has also been shown to reduce prostate cancer while maintaining the rate of detection, and patients should be informed of this fact while also indicating its possible (but unlikely) link to high grade prostate cancer and its unknown effect on prostate cancer mortality. Long-term safety and durability have been demonstrated in several studies with 
the most common adverse clinical effect related to sexual dysfunction. Cost models have investigated its effectiveness and found it a cost effective measure for treatment, especially for patients with moderate/severe symptoms and enlarged prostate $>30 \mathrm{~cm}^{3}$ when compared to TURP and tamsulosin.

In conclusion, finasteride represents a cost-effective means of reducing BPH progression and its complications in men with enlarged prostates $>30 \mathrm{~cm}^{3}$ while providing the additional benefits of decreased hematuria and prostate cancer rates.

\section{Disclosures}

The authors declare no conflicts of interest.

\section{References}

1. Emberton M, Cornel EB, Bassi PF, Fourcade RO, Gomez JM, Castro R. Benign prostatic hyperplasia as a progressive disease: a guide to the risk factors and options for medical management. Int J Clin Pract. 2008;62:1076-1086.

2. Russell DW, Wilson JD. Steroid 5 alpha-reductase: two genes/two enzymes. Annu Rev Biochem. 1994;63:25-61.

3. Jenkins EP, Andersson S, Imperato-McGinley J, Wilson JD, Russell DW. Genetic and pharmacological evidence for more than one human steroid 5 alpha-reductase. J Clin Invest. 1992;89:293-300.

4. Carson C, 3rd, Rittmaster R. The role of dihydrotestosterone in benign prostatic hyperplasia. Urology. 2003;61:2-7.

5. Norman RW, Coakes KE, Wright AS, Rittmaster RS. Androgen metabolism in men receiving finasteride before prostatectomy. J Urol. 1993;150:1736-1739.

6. Anderson JB, Roehrborn CG, Schalken JA, Emberton M. The progression of benign prostatic hyperplasia: examining the evidence and determining the risk. Eur Urol. 2001;39:390-399.

7. McConnell JD, Bruskewitz R, Walsh P, et al. The effect of finasteride on the risk of acute urinary retention and the need for surgical treatment among men with benign prostatic hyperplasia. Finasteride Long-Term Efficacy and Safety Study Group. N Engl J Med. 1998;338:557-563.

8. Gormley GJ, Stoner E, Bruskewitz RC, et al. The effect of finasteride in men with benign prostatic hyperplasia. The Finasteride Study Group. N Engl J Med. 1992;327:1185-1191.

9. Campbell MF, Wein AJ, Kavoussi LR. Campbell-Walsh Urology. Alan J. Wein AJ, editor-in-chief, Kavoussi LR, et al. eds. 9th ed. Philadelphia: W.B. Saunders; 2007.

10. Finasteride (MK-906) in the treatment of benign prostatic hyperplasia. The Finasteride Study Group. Prostate. 1993;22:291-299.

11. Andersen JT, Ekman P, Wolf H, et al. Can finasteride reverse the progress of benign prostatic hyperplasia? A two-year placebo-controlled study. The Scandinavian BPH Study Group. Urology. 1995;46:631-637.

12. Boyarsky S, Jones G, Paulson DF, Prout GR Jr. A new look at bladder neck obstruction by the food and drug administration regulators: guide lines for investigation of benign prostatic hypertrophy. Trans Am Assoc Genitourin Surg. 1976;68:29-32.

13. Bolognese JA, Kozloff RC, Kunitz SC, Grino PB, Patrick DL, Stoner E. Validation of a symptoms questionnaire for benign prostatic hyperplasia. Prostate. 1992;21:247-254.

14. Stoner E. Three-year safety and efficacy data on the use of finasteride in the treatment of benign prostatic hyperplasia. Urology. 1994;43: 284-292; discussion 92-94.

15. Hudson PB, Boake R, Trachtenberg J, et al. Efficacy of finasteride is maintained in patients with benign prostatic hyperplasia treated for 5 years. The North American Finasteride Study Group. Urology. 1999;53:690-695.
16. Boyle P, Gould AL, Roehrborn CG. Prostate volume predicts outcome of treatment of benign prostatic hyperplasia with finasteride: metaanalysis of randomized clinical trials. Urology. 1996;48:398-405.

17. Tammela TL, Kontturi MJ. Urodynamic effects of finasteride in the treatment of bladder outlet obstruction due to benign prostatic hyperplasia. J Urol. 1993;149:342-344.

18. Tammela TL, Kontturi MJ. Long-term effects of finasteride on invasive urodynamics and symptoms in the treatment of patients with bladder outflow obstruction due to benign prostatic hyperplasia. J Urol. 1995;154:1466-1469.

19. Puchner PJ, Miller MI. The effects of finasteride on hematuria associated with benign prostatic hyperplasia: a preliminary report. J Urol. 1995; 154:1779-1782.

20. Miller MI, Puchner PJ. Effects of finasteride on hematuria associated with benign prostatic hyperplasia: long-term follow-up. Urology. 1998;51:237-240.

21. Carlin BI, Bodner DR, Spirnak JP, Resnick MI. Role of finasteride in the treatment of recurrent hematuria secondary to benign prostatic hyperplasia. Prostate. 1997;31:180-182.

22. Foley SJ, Soloman LZ, Wedderburn AW, et al. A prospective study of the natural history of hematuria associated with benign prostatic hyperplasia and the effect of finasteride. J Urol. 2000;163:496-498.

23. Donohue JF, Hayne D, Karnik U, Thomas DR, Foster MC. Randomized, placebo-controlled trial showing that finasteride reduces prostatic vascularity rapidly within 2 weeks. BJU Int. 2005;96:1319-1322.

24. Memis A, Ozden C, Ozdal OL, Guzel O, Han O, Seckin S. Effect of finasteride treatment on suburethral prostatic microvessel density in patients with hematuria related to benign prostate hyperplasia. Urol Int. 2008;80:177-180.

25. Ozdal OL, Ozden C, Benli K, Gokkaya S, Bulut S, Memis A. Effect of short-term finasteride therapy on peroperative bleeding in patients who were candidates for transurethral resection of the prostate (TUR-P): a randomized controlled study. Prostate Cancer Prostatic Dis. 2005;8:215-218.

26. Mondaini N, Gontero P, Giubilei G, et al. Finasteride $5 \mathrm{mg}$ and sexual side effects: how many of these are related to a nocebo phenomenon? J Sex Med. 2007;4:1708-1712.

27. Moinpour CM, Darke AK, Donaldson GW, et al. Longitudinal analysis of sexual function reported by men in the Prostate Cancer Prevention Trial. J Natl Cancer Inst. 2007;99:1025-1035.

28. Amory JK, Anawalt BD, Matsumoto AM, et al. The effect of 5 alphareductase inhibition with dutasteride and finasteride on bone mineral density, serum lipoproteins, hemoglobin, prostate specific antigen and sexual function in healthy young men. J Urol. 2008;179:2333-2338.

29. Rahimi-Ardabili B, Pourandarjani R, Habibollahi P, Mualeki A. Finasteride induced depression: a prospective study. BMC Clin Pharmacol. 2006;6:7.

30. Lepor H, Williford WO, Barry MJ, et al. The efficacy of terazosin, finasteride, or both in benign prostatic hyperplasia. Veterans Affairs Cooperative Studies Benign Prostatic Hyperplasia Study Group. N Engl J Med. 1996;335:533-539.

31. Lepor H, Williford WO, Barry MJ, Haakenson C, Jones K. The impact of medical therapy on bother due to symptoms, quality of life and global outcome, and factors predicting response. Veterans Affairs Cooperative Studies Benign Prostatic Hyperplasia Study Group. J Urol. 1998;160:1358-1367.

32. Kirby RS, Roehrborn C, Boyle P, et al. Efficacy and tolerability of doxazosin and finasteride, alone or in combination, in treatment of symptomatic benign prostatic hyperplasia: the Prospective European Doxazosin and Combination Therapy (PREDICT) trial. Urology. 2003;61:119-126.

33. Debruyne FM, Jardin A, Colloi D, et al. Sustained-release alfuzosin, finasteride and the combination of both in the treatment of benign prostatic hyperplasia. European ALFIN Study Group. Eur Urol. 1998;34:169-175.

34. McConnell JD, Roehrborn CG, Bautista OM, et al. The long-term effect of doxazosin, finasteride, and combination therapy on the clinical progression of benign prostatic hyperplasia. N Engl J Med. 2003;349:2387-2398. 
35. Kaplan SA, McConnell JD, Roehrborn CG, et al. Combination therapy with doxazosin and finasteride for benign prostatic hyperplasia in patients with lower urinary tract symptoms and a baseline total prostate volume of $25 \mathrm{ml}$ or greater. J Urol. 2006;175:217-220; discussion 20-1.

36. Barkin J, Guimaraes M, Jacobi G, Pushkar D, Taylor S, van Vierssen Trip OB. Alpha-blocker therapy can be withdrawn in the majority of men following initial combination therapy with the dual 5 alphareductase inhibitor dutasteride. Eur Urol. 2003;44:461-466.

37. Guess HA, Heyse JF, Gormley GJ. The effect of finasteride on prostatespecific antigen in men with benign prostatic hyperplasia. Prostate. 1993;22:31-37.

38. Andriole GL, Guess HA, Epstein JI, et al. Treatment with finasteride preserves usefulness of prostate-specific antigen in the detection of prostate cancer: results of a randomized, double-blind, placebo-controlled clinical trial. PLESS Study Group. Proscar Long-term Efficacy and Safety Study. Urology. 1998;52:195-201; discussion 2.

39. Thompson CA. Finasteride may prevent prostate cancer. Am J Health Syst Pharm. 2003;60:1511, 1515.

40. Lucia MS, Epstein JI, Goodman PJ, et al. Finasteride and high-grade prostate cancer in the Prostate Cancer Prevention Trial. J Natl Cancer Inst. 2007;99:1375-1383.

41. Redman MW, Tangen CM, Goodman PJ, Lucia MS, Coltman CA, Jr, Thompson IM. Finasteride does not increase the risk of high-grade prostate cancer: a bias-adjusted modeling approach. Cancer Prev Res (Phila Pa). 2008;1:174-181.
42. Lucia MS, Darke AK, Goodman PJ, et al. Pathologic characteristics of cancers detected in The Prostate Cancer Prevention Trial: implications for prostate cancer detection and chemoprevention. Cancer Prev Res (Phila Pa). 2008;1:167-173.

43. Wilt TJ, MacDonald R, Hagerty K, Schellhammer P, Kramer BS. Fivealpha-reductase Inhibitors for prostate cancer prevention. Cochrane Database Syst Rev. 2008:CD007091.

44. Kramer BS, Hagerty KL, Justman S, et al. Use of 5 alpha-reductase inhibitors for prostate cancer chemoprevention: American Society of Clinical Oncology/American Urological Association 2008 Clinical Practice Guideline. J Urol. 2009;181:1642-1657.

45. Johansen TE, Istad JA. Long-term cost analysis of treatment options for benign prostatic hyperplasia in Norway. Scand J Urol Nephrol. 2007;41:124-131

46. Lafuma ASTP, Briand Y, Guelfucci F. Cost-efficacy of finasteride, doxazosin, and the combination of both in the treatment of benign prostatic hyperplasia (BPH) in france based on the mtops study. Value Health. 2006;9:A386-A387.

47. Svatek RS, Lee JJ, Roehrborn CG, Lippman SM, Lotan Y. Costeffectiveness of prostate cancer chemoprevention: a quality of life-years analysis. Cancer. 2008;112:1058-1065.
Therapeutics and Clinical Risk Management

\section{Publish your work in this journal}

Therapeutics and Clinical Risk Management is an international, peerreviewed journal of clinical therapeutics and risk management, focusing on concise rapid reporting of clinical studies in all therapeutic areas, outcomes, safety, and programs for the effective, safe, and sustained use of medicines. This journal is indexed on PubMed Central, CAS,

\section{Dovepress}

EMBase, Scopus and the Elsevier Bibliographic databases. The manuscript management system is completely online and includes a very quick and fair peer-review system, which is all easy to use. Visit http://www.dovepress.com/testimonials.php to read real quotes from published authors.

Submit your manuscript here: http://www.dovepress.com/therapeutics-and-clinical-risk-management-journal 